\title{
STRUCTURE-BASED DOCKING STUDIES TOWARD EXPLORING THE POTENTIAL ANTICANCER ACTIVITY OF MORIN AGAINST NON-MELANOMA SKIN CANCER THERAPEUTIC DRUG TARGETS
}

\author{
ANJUGAM C ${ }^{1}$, SRIDEVI M², GNANENDRA TS ${ }^{3 *}$ \\ ${ }^{1}$ Department of Biochemistry, Vinayaka Mission's Kirupananda Variyar Medical College and Hospitals, Vinayaka Missions Research \\ Foundation, Salem, Tamil Nadu, India. 'Department of Biotechnology, Vinayaka Mission's Kirupananda Variyar Engineering College, \\ Vinayaka Missions Research Foundation, Periyaseeragapadi, Salem, Tamil Nadu, India. ${ }^{3}$ Department of Biotechnology, Mahendra Arts and \\ Science College (Autonomous), Kalippatti, Namakkal, Tamil Nadu, India. Email: drsridevimuruhan@gmail.com
}

Received: 13 October 2017, Revised and Accepted: 14 December 2017

ABSTRACT

Objective: The purpose of this study is to explore the anticancer activity of morin compound against human cyclooxygenase-2 (COX-2) and peroxisomeproliferator-activated receptors (PPARs) isotypes (PPAR $\alpha$ and PPAR $\gamma$ ) through in silico molecular docking studies.

Methods: The 3D structures of human COX-2 complexed with ibuprofen (PDB ID: 4PH9), PPAR $\alpha$ complexed with a synthetic agonist (2S)-2-(4methoxy-3-\{[(pyren-1-yl carbonyl) amino] methyl $\}$ benzyl) butanoic acid (PDB ID: 3VI8) and PPAR $\gamma$ complexed indomethacin (PDB ID: 3ADX) were retrieved from protein databank. The cocrystallized sites were considered as binding sites, and the docking with morin compound was performed along with their respective cocrystals for each target and compared their interactions and binding affinities.

Results: It is observed that the morin compound exhibited better binding energy of $-32.9528 \mathrm{~kJ} / \mathrm{mol}$ against PPAR $\alpha$ followed by COX-2 (binding energy: $-18.4311 \mathrm{~kJ} / \mathrm{mol}$ ) and PPAR $\gamma$ (binding energy: $-17.4228 \mathrm{~kJ} / \mathrm{mol}$ ) when compared to their cocrystallized ligands.

Conclusion: The present study suggests that morin compound might serve as potential alternatives in the prevention of skin cancers by showing better activity against PPAR $\alpha$.

Keywords: Non-melanoma skin cancer, Cyclooxygenase-2, Peroxisome-proliferator-activated receptors, Molecular docking, Morin.

(C) 2018 The Authors. Published by Innovare Academic Sciences Pvt Ltd. This is an open access article under the CC BY license (http://creativecommons. org/licenses/by/4. 0/) DOI: http://dx.doi.org/10.22159/ajpcr.2018.v11i4.23076

\section{INTRODUCTION}

In general, the carcinogenesis is a multistage process as initiation, promotion, and progression that depends on various susceptibility factors including the exposure of oncogenes and tumor suppressor genes to carcinogens [1,2]. Many studies on the mouse skin carcinogenesis models have provided the better understanding of cellular and molecular events that are involved in the initiation, promotion, and progression [3-6]. Furthermore, these studies have provided an ample evidence of various chemical and physical agents including greater exposure to ultraviolet (UV) radiations from sunlight can cause skin cancers. Nowadays, almost $40 \%$ of newly diagnosed cancers patients across the globe are reported with skin cancers [7]. These dramatic increases in the incidence of worldwide skin cancer are due to the increased rate of ozone depletion, which has significantly resulted in increased earth surface UV radiation intensity.

Over the years, the increased UV-B radiations resulted in UV radiationinduced gene mutations, which have significant effects on skin carcinogenesis and on the rate of increased incidence in skin cancer cases that have been recognized as a serious public health issue [8]. At present, skin cancer is growing as a dreadful human disease as compared to other cancers. The two major categories of skin care are melanocytic (melanomas) and epithelial (non-melanoma) skin cancers. Furthermore, this non-melanoma skin cancer (NMSC) is categorized into basal cell carcinomas with slow growth and rare metastasis and squamous cell carcinomas with strong invasive and metastasis characteristics [9]. Above all, melanomas (melanocytic skin cancer) were being the main cause of deaths in skin cancer patients, which accounts for only $4 \%$ of skin cancers [10]. However, the mainstay of skin cancer prevention has focused on advising people to apply sunscreens regularly.

Furthermore, many studies on the mechanisms of antitumor initiating and antitumor-promoting properties of potent drugs are currently being suggested for the prevention of skin cancer and other epithelial cancers in humans [11]. In line with this, various natural and dietary agents have been identified as potent chemopreventive agents for UV-induced skin cancers. However, many of these natural and dietary compounds contain polyphenols that have a variety of different activities [12]. In this scenario, many recent studies have suggested that proanthocyanidins can inhibit the cyclooxygenase-2 (COX-2) and peroxisome-proliferator-activated receptors (PPARs) expression and put forth that their expression levels are associated with the reduction of UV-induced skin tumors in mice.

The PPARs are ligand-activated transcription factors that belong to the nuclear-hormone-receptor family. Among the three isotypes (PPAR $\alpha$, $\operatorname{PPAR} \beta / \delta$, and PPAR $\gamma$ ), PPAR $\alpha$ is the therapeutic target of hypolipidemic compounds that are used in the treatment of dyslipidemia. While $\operatorname{PPAR} \beta / \delta$ and PPAR $\gamma$ are targets of the thiazolidinediones and Type II diabetes. Furthermore, these PPAR isotypes are associated with pathways including lipid metabolism, skin homeostasis, and carcinogenesis; they are likely considered as potential therapeutic targets of skin cancers [13-15]. On the other hand, COX catalyze the formation of prostaglandins from arachidonic acid. The major COX isoforms are COX-1 and COX-2. The increased level of prostaglandinendoperoxide synthases (PEG2) increase with the UV-induced COX2 expression and results in progression of NMSC $[16,17]$. Thus, the 
present study was undertaken to explore the anticancer activity of morin compounds through revealing its binding efficacy against the three selected therapeutic drug targets of skin cancer using in silico molecular docking methods.

\section{METHODS}

\section{Target selection}

The cocrystallized X-ray structure of human COX-2 complexed with Ibuprofen (PDB ID: 4PH9), PPAR $\alpha$ complexed with a synthetic agonist (2S)-2-(4-methoxy-3-\{[(pyren-1-yl carbonyl) amino] methyl benzyl) butanoic acid (PDB ID: 3VI8) and PPAR $\gamma$ complexed indomethacin, and nitro-233 (PDB ID: 3ADX) were retrieved from protein databank [18].

\section{Ligand selection}

The 3D structures of Ibuprofen (PubChem ID: 3672), Indomethacin (PubChem ID: 3715) and morin (PubChem ID: 5281670) were retrieved as SDF format from PubChem database [19]. While the 2D structure of (2S)-2-(4-methoxy-3-\{[(pyren-1-ylcarbonyl) amino] methyl $\}$ benzyl) butanoic acid (APHM13) compound was drawn in ACD Chemsketch [20] and obtained its SMILES notation and the 3D was generated and converted into SDF format using "Online SMILES convertor and Structure file generator" server [21]

\section{Binding site prediction}

The amino acid residues in binding sites of Human COX-2, PPAR $\alpha$, and PPAR $\gamma$ are defined using the reference Ligands such as ibuprofen, (2S)2-(4-methoxy-3-\{[(pyren-1-ylcarbonyl)amino]methyl\}benzyl)butanoic acid and indomethacin, respectively. The amino acid residues within 6 $\AA$ radius of these reference Ligand were included while selecting the binding site from all the three receptors for docking studies with morin using LeadIT (Version 2.1.9) [22].

\section{Molecular docking studies}

The 3D structures ibuprofen, (2S)-2-(4-methoxy-3-\{[(pyren-1ylcarbonyl) amino] methyl b benzyl) butanoic acid and indomethacin were docked within the binding pockets of Human COX-2, PPAR $\alpha$ and PPARy, respectively, using FlexX module of LeadIT. While the 3D structure of morin was used to virtually screen against the selected three targets. The docking was performed with the default parameters such as triangle matching base placements, zero full score and no score contributions and threshold for full score and no score contributions of 30 and 70, respectively, clash handling values of 2.9 Åand 0.6 for protein-ligand clashes and intraligand clash factors and maximum number of solutions per iteration being 200 as the default docking values and also per fragmentations [23].

\section{Docking interactions}

The docking interactions revealing the formation of H-bond and Non-bonded contacts among the ligand compounds and the binding site amino acid residues of selected targets were analyzed using poseview [24] module of LeadIT.

\section{RESULTS AND DISCUSSION}

The majority of skin cancers are caused by overexposure to ultraviolet radiation from the sun and artificial light sources including tanning bed usages. However, the mainstays for the prevention of skin cancer are focused on avoidance of exposure to excessive sun UV radiations and recommendations for the usage of protective hats and longsleeved clothing $[25,26]$. However, there is no effect of sunscreens on prior UV damage to the skin. In this scenario, the needs for additional measures are required to retard the rising incidence of NMSC. Thus, the identification of more and adequate potential drug agents targeting against novel therapeutic drug targets of skin cancer might serve as potential alternatives in the prevention of skin cancers. In this context, many pharmaceutical industries are trying hard to put forth the potential drug compounds through the available computational strategies such as structure-based drug designing in particular molecular docking studies. A huge number of reports available on the significance of molecular docking studies in the screening of potential novel compounds and development of drugs against various diseases [27-29]. Thus, in the present study, we have explored the anticancer activity of morin compound and its binding efficiency through docking studies against three potential drug targets of NMSC.

\section{Target selection}

Considering that the increased level of PEG2 synthases with the increase in UV-induced COX-2 expression and its association in progression of NMSC was considered as one of the three selected therapeutic targets in this study, while the PPAR isotypes such as PPAR $\alpha$ and PPAR $\gamma$ were considered as other two therapeutic targets as they are associated with pathways including lipid metabolism, skin homeostasis, and carcinogenesis. The 3D structures of these targets such as human COX-2 complexed with ibuprofen (PDB ID: 4PH9), PPAR $\alpha$ complexed with a synthetic agonist (2S)-2-(4-methoxy-3$\{[$ (pyren-1-ylcarbonyl) amino]methyl $\}$ benzyl) butanoic acid (PDB ID: 3 VI8) and PPAR $\gamma$ complexed indomethacin, and Nitro-233 (PDB ID: 3ADX) (Fig. 1a-c) were retrieved from Protein Databank and used as receptor for further docking studies.

\section{Ligands}

The 3D structure of four ligands such as Ibuprofen, (2S)-2-(4-methoxy-3$\{[($ pyren-1-ylcarbonyl)amino]methyl\}benzyl) butanoic acid (APHM13), indomethacin and morin compounds along with their 2D structures, IUPAC names, molecular formulae and molecular weight were given in Table 1.

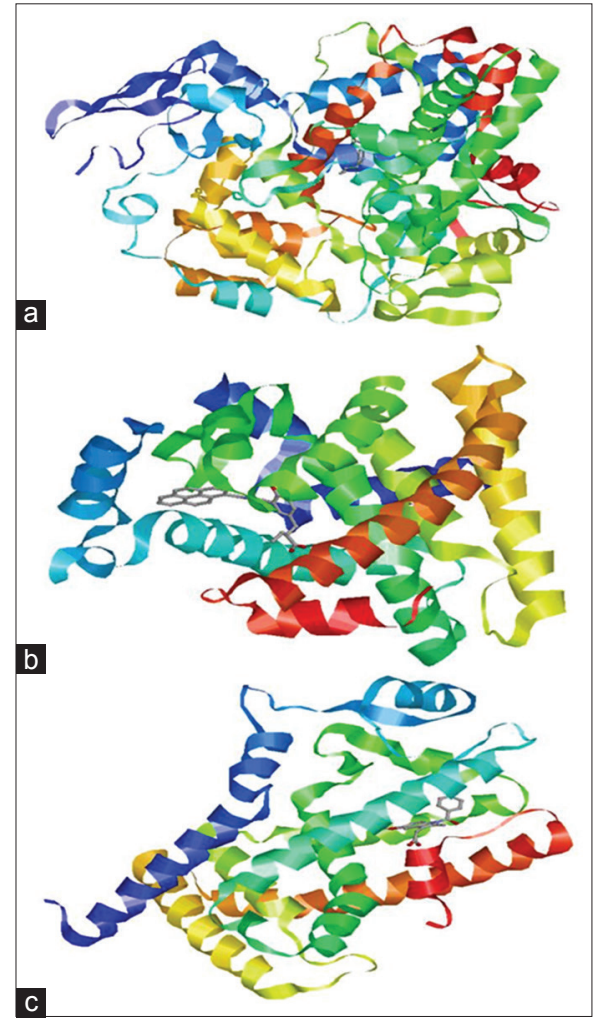

Fig. 1: The 3D structures of selected potential therapeutic drug targets for skin cancer represented in ribbons from with group colors using rasmol (a) human cyclooxygenase- 2 complexed with ibuprofen (PDB ID: 4PH9), (b) peroxisome-proliferator-activated receptors alpha (PPAR $\alpha$ ) complexed with a synthetic agonist (2S) 2-(4-methoxy-3-\{[(pyren-1-yl carbonyl) amino] methyl\} benzyl) butanoic acid (PDB ID: 3VI8)' (c) peroxisome-proliferatoractivated receptors gamma (PPAR $\gamma$ ) complexed indomethacin (PDB ID: 3ADX) 
Among these ligands, ibuprofen, (2S)-2-(4-methoxy-3-\{[(pyren-1ylcarbony l) amino] methyl benzyl) butanoic acid and indomethacin were used as reference ligands for human COX-2, PPAR $\alpha$, and PPAR $\gamma$, respectively. While the morin structure was used to dock within all the three receptors to reveal its anticancer activity against these novel therapeutic targets of skin cancer.

\section{Virtual screening}

It is observed that morin exhibited theoretically encouraging docking scores. However, the docking scores of morin in comparison with the reference ligand binding energy of respective selected drug targets were found to be low. It is observed that the better binding energy of $-32.9528 \mathrm{~kJ} / \mathrm{mol}$ was exhibited against the therapeutic target, PPAR $\alpha$ followed by COX-2 (binding energy: $-18.4311 \mathrm{~kJ} / \mathrm{mol}$ ) and PPAR $\gamma$ (binding energy: $-17.4228 \mathrm{~kJ} / \mathrm{mol}$ ). Thus, suggesting that morin compound might possess better anticancer activity against PPAR $\alpha$.

\section{Docking interactions of COX-2}

The docking interactions of cocrystallized ligand, Ibuprofen with human COX-2 exhibited the better docking interactions with a binding energy of $-20.5529 \mathrm{~kJ} / \mathrm{mol}$. This interaction is favored by the formation of H-bond with Arg121 and hydrophobic interactions with Val117, Val350, Leu353, Ser354, Tyr356, Phe519, Leu532, Val524, and Als528. Whereas, the docking interactions of morin are observed with a binding energy of $-18.4311 \mathrm{~kJ} / \mathrm{mol}$. This interaction is supported by the formation of Hbonds with Leu353, Ser354, Met523, and Ser531 and non-bonded interactions with Leu353, Ser354, Tyr356, Tyr386, Trp388, Phe519, Val524, and Gly527. The docking complex and interaction plots of binding interactions of cocrystallized ligand, Ibuprofen and morin with Human COX-2 shown in Fig. 2. It is observed that the amino acid Arg121 in Human COX-2 is crucial in favoring the interactions with cocrystallized ligand, Ibuprofen. While the amino acid Met523 and Ser531 are vital for interactions with morin, which has significantly reflected the different binding energies.

\section{Docking interactions of PPARo}

The docking interactions of cocrystallized ligand, (2S)-2-(4-methoxy$3-\{[($ pyren-1-ylcarbony l) amino] methyl $\}$ benzyl) butanoic acid with
PPAR $\alpha$ showed the binding energy of $-41.209 \mathrm{~kJ} / \mathrm{mol}$. This interaction is favored by the formation of H-bond with Cys276, Ser280, Tyr314, Tyr464, and His440 along with Hoh1060 and hydrophobic interactions with Cys275, Ile339, Val332, Val255, Met330, Ile272, Ile241, Ala333, Thr279, Leu247, Gln277, Ile354, Tyr314, Ser280, Met355, Leu321, Phe318, His440, and Cys276 whereas, the docking interactions of morin exhibited the binding energy of $-32.9528 \mathrm{~kJ} / \mathrm{mol}$. This interaction is supported by the formation of Hbonds with Met330, Ile354, Tyr314, and Hoh1026 and non-bonded interactions with Met325, Lys358, Ile354, Cys276, Met355, Phe318, Leu321, Gly277, Tyr314, Val444, Ser280, and His440. The docking complex and interaction plots of binding interactions of cocrystallized ligand, (2S)-2-(4-methoxy-3$\{[($ pyren-1-ylcarbony l) amino] methyl $\}$ benzyl) butanoic acid and morin with PPAR $\alpha$ shown in Fig. 3.

It is observed that amino acid Tyr314 and sulfur containing amino acids such as cys 276 and met325 in the binding sites of PPAR $\alpha$ were crucial for the interactions with cocrystallized ligand, (2S)-2-(4-methoxy$3-\{[$ (pyren-1-ylcarbony l) amino] methyl $\}$ benzyl) butanoic acid and morin, respectively. Furthermore, the difference in amino acids favoring $\mathrm{H}$-bond and non-bonded interaction with cocrystallized ligand, (2S)-2(4-methoxy-3-\{[(pyren-1-ylcarbony l) amino] methyl $\}$ benzyl) butanoic acid and morin have significantly affected the binding energies.

\section{Docking interactions of PPAR $\gamma$}

The docking interactions of cocrystallized ligand, Indomethacin with PPAR $\gamma$ showed the binding energy of $-25.3056 \mathrm{~kJ} / \mathrm{mol}$. This interaction is favored by the formation of H-bond with Tyr473, His449, and His323 and hydrophobic interactions with Phe282, Phe363, Phe360, Ile281, Leu469, Gln286, Met364, Cys285, Lys367, and His449 whereas, the docking interaction of morin exhibited the binding energy of $-17.4228 \mathrm{~kJ} / \mathrm{mol}$. This interaction is supported by the formation of Hbonds with Ser289, Met364, Ala278, and Gln286 and non-bonded interactions with Ile281, Phe282, Phe363, Phe360, Cys285, Leu465, Gln286, and His449. The docking complex and interaction plots of binding interactions of cocrystallized ligand, Indomethacin and morin with PPARy were shown in Fig. 4. It is observed that completely

Table 1: Selected phytochemical compounds and their plant sources

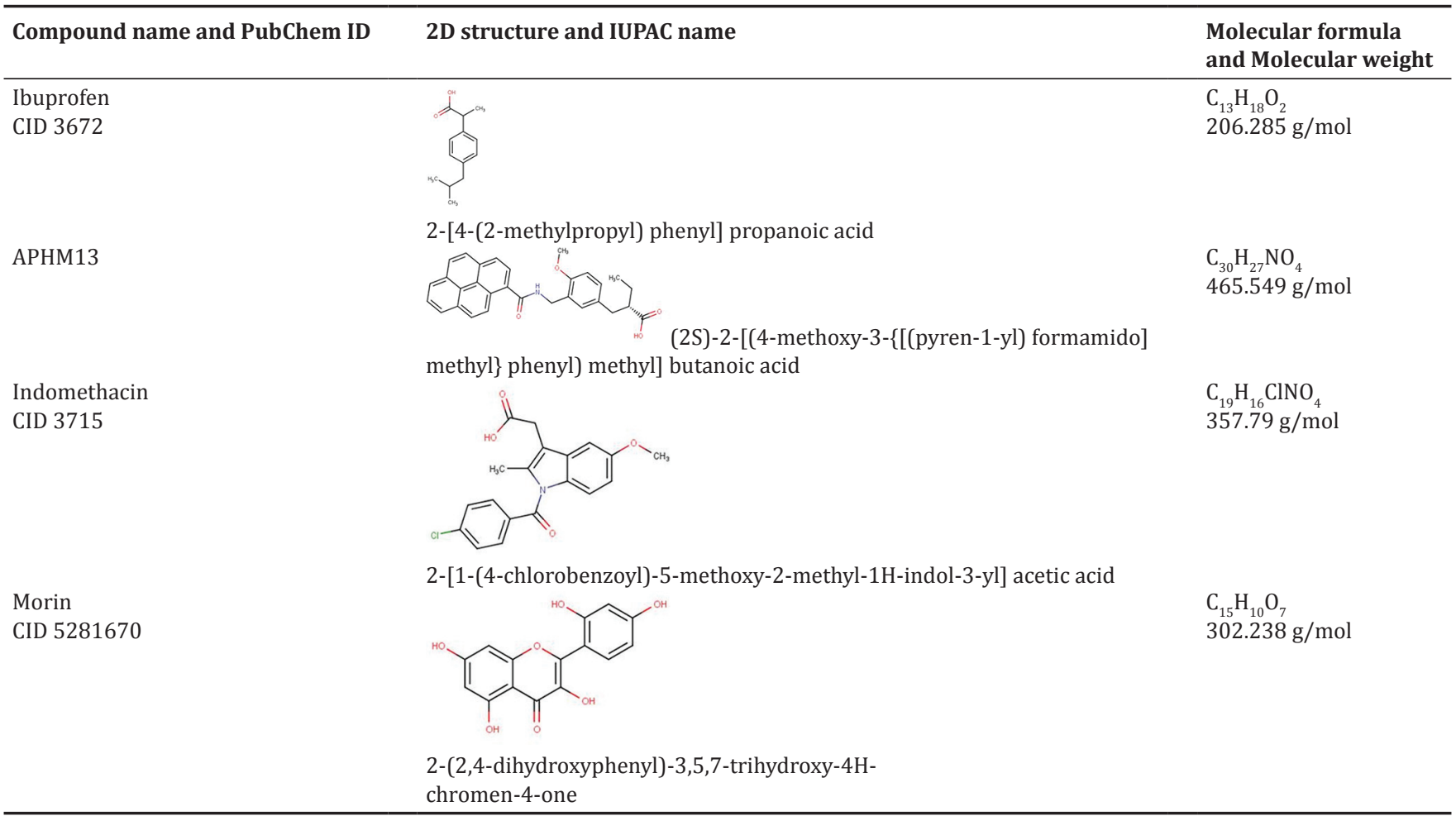




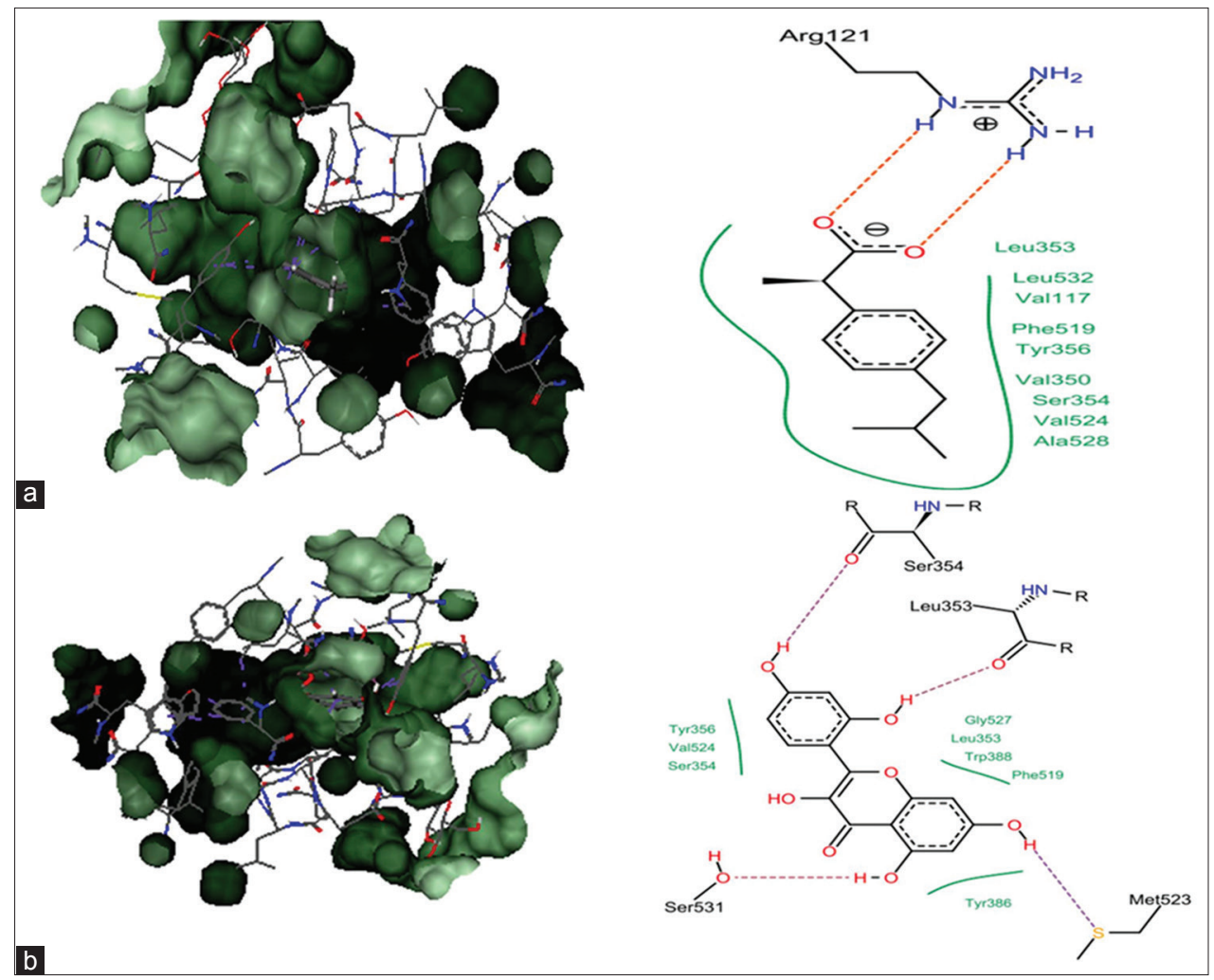

Fig. 2: Docking complex and interactions of cyclooxygenase-2, (a) cocrystallized ligand, Ibuprofen docking interactions with a binding energy of $-20.5529 \mathrm{~kJ} / \mathrm{mol}$, (b) docking interactions of morin with a binding energy of $-18.4311 \mathrm{~kJ} / \mathrm{mol}$

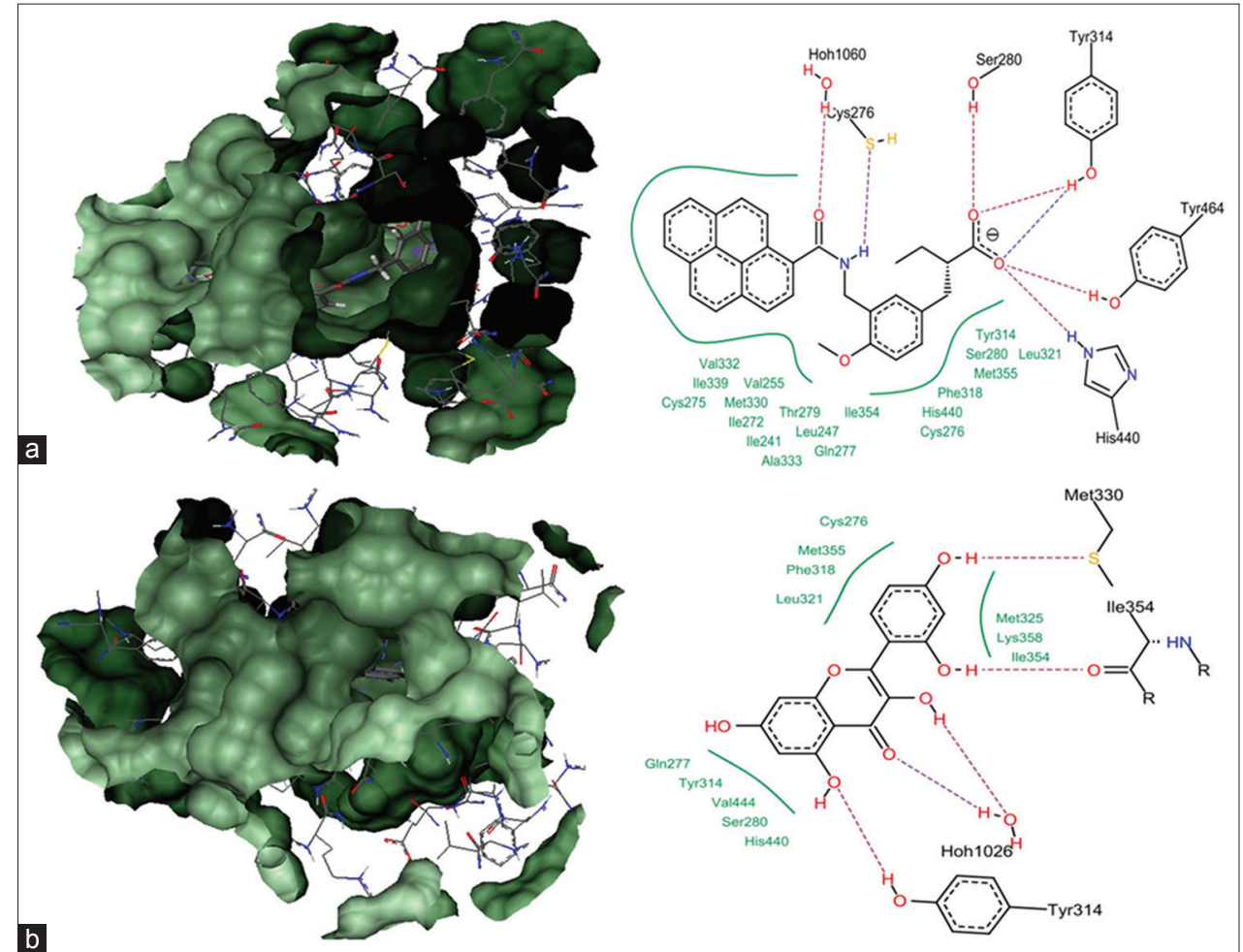

Fig. 3: Docking complex and interactions of peroxisome-proliferator-activated receptors alpha, (a) cocrystallized ligand, (2S)-2-(4methoxy-3-\{[(pyren-1-ylcarbony l) amino] methyl benzyl) butanoic acid docking interactions with binding energy of $-41.209 \mathrm{~kJ} / \mathrm{mol}$, (b) docking interactions of morin with binding energy of $-32.9528 \mathrm{~kJ} / \mathrm{mol}$

different amino acid in the binding sites of PPAR $\gamma$ was crucial for the $\mathrm{H}$-bond interactions with cocrystallized ligand Indomethacin and morin which significantly revealed the difference in their binding energies. 


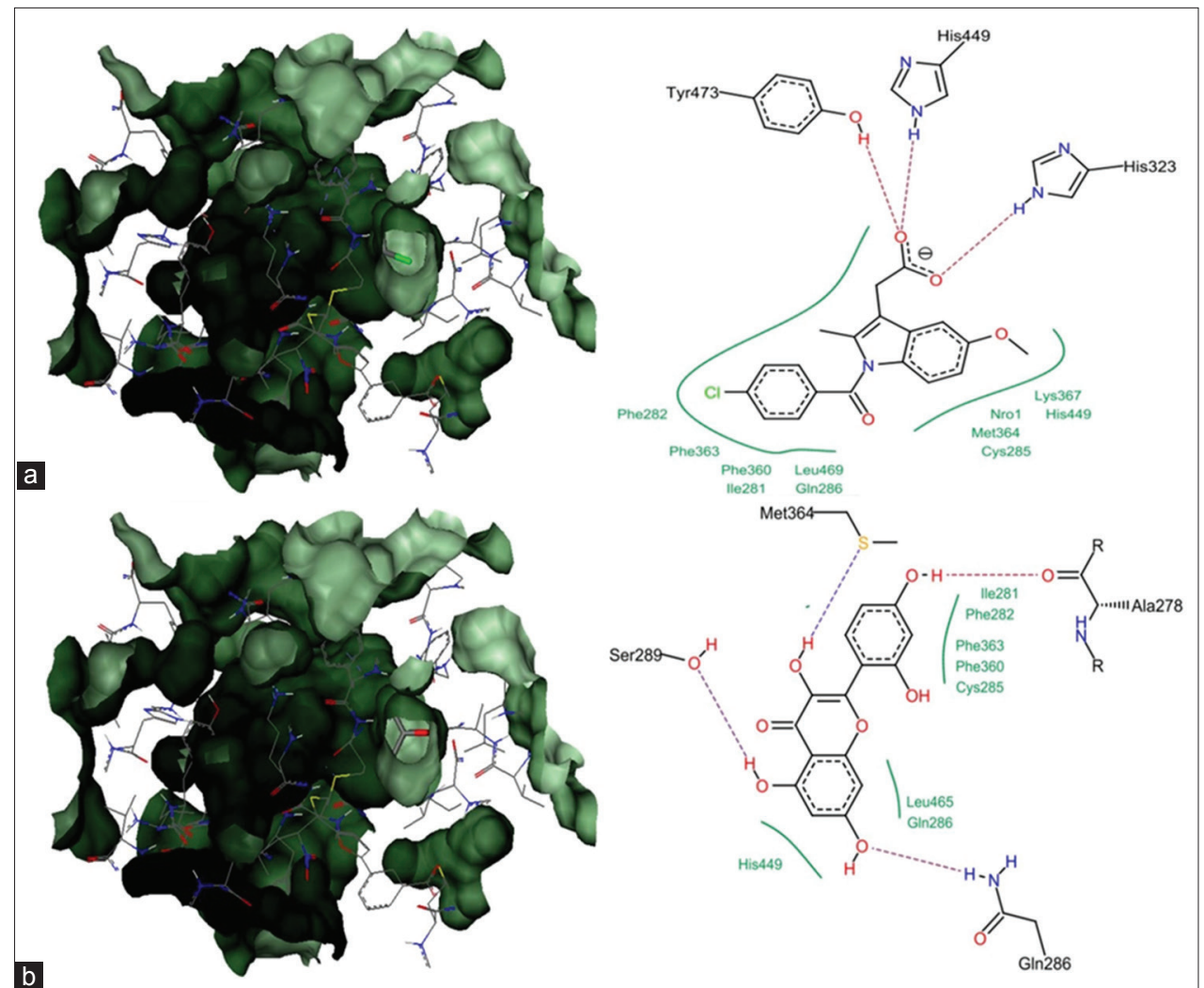

Fig. 4: Docking complex and interactions of peroxisome-proliferator-activated receptors alpha, (a) cocrystallized ligand, Indomethacin docking interactions with binding energy of $-25.3056 \mathrm{~kJ} / \mathrm{mol}$, (b) docking interactions of morin with binding energy of $-17.4228 \mathrm{~kJ} / \mathrm{mol}$

However, considering the binding energies of morin when compared to the cocrystallized ligands and the vast difference in the amino acids within the binding sites of selected targets such as human COX-2, PPAR $\alpha$, and PPAR $\gamma$ favoring the H-bond interactions, suggests that morin might possess different activities against the targets when compared to their respective cocrystals. Thus, we emphasize that morin compound might possess better anticancer activity against these selected skin cancer therapeutic targets, while it possesses better activity against PPAR $\alpha$ based on its binding energies.

Furthermore, these docking studies of cocrystallized ligands with chosen protein targets implies that the presence of keto groups $(=0)$ and amide groups $(-\mathrm{N}=\mathrm{H})$ as electronegative elements on the compound were crucial for interactions with binding site residues. While it is observed that presence of keto groups $(=0)$ on morin compounds plays a significant role for its activity against these three selected targets. Interestingly it is observed that $\mathrm{OH}$ group on morin has showed bonding interactions with sulfur-containing amino acid methionine in all the three targets. Therefore, these prioritized drug targets and drug compounds interactions might play a fundamental role in the expansion of new and urgently-needed drugs for the prevention of UVinduced progression of NMSC.

\section{CONCLUSION}

As the expression levels of COX-2 and PPARs are associated with the reduction of UV-induced skin carcinogenesis, in the present study, the anticancer activity of morin compound was put forth as a potential drug agent by targeting these COX-2 and PPAR $\alpha$ and PPAR $\gamma$ isotypes through structure-based drug designing. Furthermore, the study has emphasizes that morin compound might possess better anticancer activity against these selected skin cancer therapeutic targets based on its binding energies. The morin compound has shown better activity against PPAR $\alpha$ followed by COX-2 PPAR $\gamma$, and furthermore, the understanding of docking interactions has revealed the importance of electronegative elements on the compounds and their interactions with sulfur-containing amino acid methionine in all the three targets. Thus, the study has significantly revealed the anticancer activity of morin and the role of COX-2 and PPAR $\alpha$ and PPAR $\gamma$ isotypes as prioritized drug targets of skin cancer.

\section{AUTHORS CONTRIBUTIONS}

The first author carried out of the experimental part of the work. The second and third authors guided and monitored the experimental design, data compilation, and corrected the manuscript.

\section{CONFLICT OF INTERESTS}

The authors do not have any conflict of interest to declare.

\section{REFERENCES}

1. Lanoy E. Epidemiology, risk factor and screening for melanoma and other skin cancers. Rev Prat 2014;64:31-6.

2. Bulliard JL, Panizzon RG, Levi F. Epidemiology of epithelial skin cancers. Rev Med Suisse 2009;5:882-4.

3. Fischer SM, Hawk ET, Lubet RA. Coxibs and other nonsteroidal antiinflammatory drugs in animal models of cancer chemoprevention. Cancer Prev Res (Phila) 2011;4:1728-35.

4. Kripke ML. Antigenicity of murine skin tumors induced by ultraviolet light. J Natl Cancer Inst 1974;53:1333-6.

5. Rogers HW, Coldiron BM. Analysis of skin cancer treatment and costs in the United States Medicare population, 1996-2008. Dermatol Surg 2013;39:35-42.

6. Rogers HW, Weinstock MA, Harris AR. Incidence estimate of nonmelanoma skin cancer in the United States, 2006. Arch Dermatol 2010;146:283-7.

7. Elmets CA, Ledet JJ, Athar M. Cyclooxygenases: Mediators of UVinduced skin cancer and potential targets for prevention. J Invest Dermatol 2014;134:2497-502

8. Shujiao L, Lilin H, Yong S. Cyclooxygenase-2 expression and 
association with skin cancer: A meta-analysis based on Chinese patients. J Can Res Ther 2016;12:288.

9. Khan AR, Sadiq IZ, Abdullahi LI, Danlami D, Taneja P. Chemoprotective role of bovine lactoferricin against 7, 12 dimethylbenz (a) anthracene induced skin cancer in female swiss albino mice. Int J Pharm Pharm Sci 2016;8:215-22.

10. Wilgus TA, Koki AT, Zweifel BS, Kusewitt DF, Rubal PA, Oberyszyn TM, et al. Inhibition of cutaneous ultraviolet light B-mediated inflammation and tumor formation with topical celecoxib treatment. Mol Carcinog 2003;38:49-58.

11. Seukeran DC, Newstead CG, Cunliffe WJ. The compliance of renal transplant recipients with advice about sun protection measures. $\mathrm{Br} \mathrm{J}$ Dermatol 1998;138:311-4.

12. John R, Cesar L, Yhors C. The health benefits of natural skin UVA photoprotective compounds found in botanical sources. Int J Pharm Pharm Sci 2016;S1:13-23.

13. Michalik L, Desvergne B, Wahli W. Peroxisome-proliferator-activated receptors and cancers: Complex stories. Nat Rev Cancer 2004;4:61-70.

14. He G, Muga S, Thuillier P, Lubet RA, Fischer SM. The effect of PPARgamma ligands on UV-or chemically-induced carcinogenesis in mouse skin. Mol Carcinog 2005;43:198-206.

15. Thuillier P, Anchiraico GJ, Nickel KP, Maldve RE, Gimenez-Conti I, Muga SJ, et al. Activators of peroxisome proliferator-activated receptoralpha partially inhibit mouse skin tumor promotion. Mol Carcinog 2000;29:134-42.

16. Brecher AR. The role of cyclooxygenase-2 in the pathogenesis of skin cancer. J Drugs Dermatol 2002;1:44-7.

17. Meng H, Zhao F, Li T, Hongying C, Liuqing C, Weizhen W. Survivin and $\mathrm{COX}-2$ expression in squamous cell skin cancer and basal cell skin Carcinoma. Chin J Dermato Venereol 2010;9:804-6.

18. Berman HM, Battistuz T, Bhat TN, Bluhm WF, Bourne PE, Burkhardt $\mathrm{K}$, et al. The protein data bank. Acta Crystallogr D Biol Crystallogr 2002;58:899-907.

19. Kim S, Thiessen PA, Bolton EE, Chen J, Fu G, Gindulyte A, et al.
PubChem substance and compound databases. Nucleic Acids Res 2016;44:D1202-13.

20. ACD/ChemSketch Freeware, version 12. Toronto, ON, Canada: Advanced Chemistry Development, Inc. Available from: http://www. acdlabs.com

21. Weininger D. SMILES, a chemical language and information system. Introduction to methodology and encoding rules. J Chem Inf Comput Sci 1998;28:31-6.

22. Rarey M, Kramer B, Lengauer T, Klebe G. A fast flexible docking method using an incremental construction algorithm. J Mol Biol 1996;261:470-89.

23. Gnanendra S, Anusuya S, Natarajan J. Molecular modeling and active site analysis of SdiA homolog, a putative quorum sensor for Salmonella typhimurium pathogenecity reveals specific binding patterns of AHL transcriptional regulators. J Mol Model 2012;18:4709-19.

24. Stierand K, Maab P, Rarey M. Molecular complexes at a glance: Automated generation of two-dimensional complex diagrams. Bioinformatics 2006;22:1710-6.

25. Thompson SC, Jolley D, Marks R. Reduction of solar keratoses by regular sunscreen use. N Engl J Med 1993;329:1147-51.

26. Ibrahem NH. Extraction and characterization of Iraqi Artemisia dracunculus dried aerial parts extract though hplc and gc-ms analysis with evaluation of its antitumor activity against 7,12-dimethylbenze(a) anthracene induced skin cancer in mice. Int $\mathrm{J}$ Pharm Pharm Sci 2017;9:34-42.

27. Doucet-Personeni C, Bentley PD, Fletcher RJ, Kinkaid A, Kryger G, Pirard B, et al. A structure-based design approach to the development of novel, reversible AChE inhibitors. J Med Chem 2001;44:3203-15.

28. Duffy BC, Zhu L, Decornez H, Kitchen DB. Early phase drug discovery: Cheminformatics and computational techniques in identifying lead series. Bioorg Med Chem 2012;20:5324-42.

29. Durrant JD, Mccammon JA. Computer-aided drug-discovery techniques that account for receptor flexibility. Curr Opin Pharmacol 2010;10:770-4. 